\title{
ON LIMITS OF SCALAR OPERATORS
}

\author{
BY \\ JAMES E. SIMPSON(1)
}

1. Introduction. The basic problem under consideration in the first part of this paper is that of determining conditions under which a weak or strong limit of scalar operators is again a scalar operator. This question has been treated by Bade [1], Foguel [7], [8], and Edwards and Ionescu Tulcea [6], among others. The central result considered here is a generalization of a theorem due to Rellich [12]. Foguel's approach is used below to obtain a variation of the previous forms of Rellich's theorem which is valid in locally convex spaces which are barreled, separated, and quasi-complete. This is the Corollary of Theorem 3 below. In the last section the connection between this theorem and Foguel's is explored. There is also included some material on Boolean algebras (B.A.'s) of projections, which generalizes many of the theorems of Bade in [1] and [2].

In the process of this work we give a generalization to locally convex spaces of a theorem of Dunford's to the effect that a bounded Boolean algebra of projections on a weakly complete space is contained in a $\sigma$-complete B.A. (which is in turn contained in the strong closure of the original B.A.). As noted in [2], the proof given in [4,p. 578], for a special case, does indeed generalize, even to the locally convex case. The proof given below, however, is somewhat different, although the essential tool, namely the family of measures constructed at the beginning of the proof, is the same.

2. Preliminary concepts. Throughout this paper $E$ will denote a locally convex linear topological space over the complex field $C$, which is separated, barreled, and quasi-complete. We denote by $E^{\prime}$ the strong dual of $E$, that is, the space of linear continuous mappings of $E$ into the complex field, endowed with the topology of uniform convergence on bounded subset of $E$. Let $\mathscr{E}$ be the family of equicontinuous subsets of $E^{\prime}$. If $F$ is any linear topological space, $L(E, F)$ will denote the space of continuous linear mappings of $E$ into $F$. The dual map of an element $U$ of $L(E, F)$ will be denoted by ${ }^{t} U$. For $Z$ a locally compact space, we denote by $\mathscr{K}(Z)$ the algebra of all complex-valued continuous functions defined on $Z$ which have compact support, and by $M^{1}(Z)$ the Banach space of

Received by the editors July 6, 1965 .

(1) Part of the contents of this paper are taken from the author's Ph. D. dissertation, Yale University. The remainder was supported by National Science Foundation research contract GP-1233. Some of the contents were presented to the Society, April 9, 1965. 
all complex bounded Radon measures on $Z$. The characteristic function of a subset $D$ of $Z$ will be denoted $\phi_{D}$. Let $B_{0}(Z)$ denote the algebra of complex-valued Baire functions, $B_{0}^{\infty}(Z)$ the bounded functions in $B_{0}(Z)$, and $S_{0}(Z)$ the Baire sets of $Z$. The symbols $B(Z), B^{\infty}(Z)$, and $S(Z)$ are similarly defined, using "Borel" instead of "Baire." A family $\mathscr{F}=\left\{m_{x, x^{\prime}}\right\}_{x \in E, x^{\prime} \in E^{\prime}}$ of bounded Radon measures on $Z$ is said to be semispectral if the mapping $h:\left(x, x^{\prime}\right) \rightarrow m_{x, x^{\prime}}$ of $E \times E^{\prime} \rightarrow M^{1}(Z)$ is bilinear and $\mathscr{E}$-hypocontinuous. $\mathscr{E}$-hypocontinuous means that $h$ is separately continuous and for every neighborhood $W$ of zero in $M^{1}(Z)$ and every set $M$ in $\mathscr{E}$ there is a neighborhood $V$ of zero in $E$ such that $h(V \times M) ! \subseteq W$. We denote by $L^{\infty}(\mathscr{F})$ the set of all complex-valued functions on $Z$ which are $m_{x, x}$-measurable for every $x \in E$ and $x^{\prime} \in E^{\prime}$, for which $N^{\infty}(f, \mathscr{F})=\inf \{\alpha>0:\{z:|f(z)|>\alpha\}$ is $m_{x, x^{\prime}}$-negligible for all $\left.x \in E, x^{\prime} \in E^{\prime}\right\}$ is finite. For fixed $x \in E, f \in L^{\infty}(\mathscr{F})$, define a mapping $U_{f} x$ of $E^{\prime}$ into $C$ by $\left\langle U_{f} x, x^{\prime}\right\rangle=\int_{z} f d m_{x, x^{\prime}}$. It follows, by reasonably straighforward calculations, using the properties of $h$, that the mapping $U_{f}: x \rightarrow U_{f} x$ has range in $E^{\prime \prime}$, is linear, and is continuous when $E^{\prime \prime}$ has the toplogy of uniform convergence on elements of $\mathscr{E}$. Also, $\left\{U_{f}: N^{\infty}(f, \mathscr{F}) \leqq 1\right\}$ is an equicontinuous subset of $L\left(E, E^{\prime \prime}\right)$. For if $A \in \mathscr{E}$ and $\varepsilon>0$, there is a neighborhood $V$ of zero in $E$ such that $\left\|m_{x, x^{\prime}}\right\| \leqq \varepsilon$ for all $x \in V, x^{\prime} \in A$. We deduce $\left|\left\langle U_{f} x, x^{\prime}\right\rangle\right| \leqq \varepsilon$ if $f \in L^{\infty}\left(\mathscr{F}, N^{\infty}(f, \mathscr{F}) \leqq 1, x \in V\right.$, and $x^{\prime} \in A$. Since the polars of the sets of $\mathscr{E}$ are a fundamental system of neighborhoods of zero in $E^{\prime \prime}$, the equicontinuity just asserted is proved. We shall assume that for all our semispectral families, the range of $U_{f}$ is in $E$ for all $f \in L^{\infty}(\mathscr{F})$, and that $U_{1}=I$, where 1 is the function $1(z)=1$ for all $z \in Z$. A semispectral family of bounded Radon measures is said to be spectral if $f \cdot m_{x, x^{\prime}}=m_{U_{f} x, x^{\prime}}=m_{x, y^{\prime}}$ where $y^{\prime}={ }^{t} U_{f} x^{\prime}$ for every $f \in L^{\infty}(\mathscr{F}), x \in E$, and $x^{\prime} \in E^{\prime}$. If $\mathscr{F}$ is spectral then the mapping $f \rightarrow U_{\mathscr{F}, f}$ is a representation of the algebra $L^{\infty}(\mathscr{F})$ into the algebra $L(E, E)$. If $\mathscr{F}$ is semispectral and $f \cdot m_{x, x^{\prime}}=m_{U_{\mathscr{F},}, x, x^{\prime}}$ for every $f \in B_{0}(Z), x \in E$, and $x^{\prime} \in E^{\prime}$, then $\mathscr{F}$ is spectral. The above material is taken essentially from [10].

Let $\mathscr{F}=\left\{m_{x, x^{\prime}}\right\}_{x \in E, x^{\prime} \in E^{\prime}}$ be a spectral family on $Z$. Define $P: S_{0}(Z) \rightarrow L(E, E)$ by the formula $P(D)=U_{\mathscr{F}, \phi_{D}}$. Then $P$ has the properties

$$
\begin{aligned}
& D \rightarrow P(D) x \text { is countably additive on } S_{0}(Z) \text { for each } x \in E ; \\
& P\left(D^{\prime} \cap D^{\prime \prime}\right)=P\left(D^{\prime}\right) P\left(D^{\prime \prime}\right) \text { for all } D^{\prime}, D^{\prime \prime} \text { in } S_{0}(Z) ; \\
& \left\{P(D): D \in S_{0}(Z)\right\} \text { is an equicontinuous subset of } L(E, E) ; \\
& P(Z)=I .
\end{aligned}
$$

Any mapping from $S_{0}(Z) \rightarrow L(E, E)$ with these four properties is called a spectral measure on $Z$. It can be shown that for every spectral measure $P$ on $Z$, there is a unique spectral family $\mathscr{F}$ on $Z$ such that $P(D)=U_{\mathscr{F}, \phi_{D}}$ for every $D$ in $S_{0}(Z)$. When $\mathscr{F}$ is a spectral family on $Z$, it will often be convenient to write $\int_{Z} f d P$ for $U_{\mathscr{F}, \mathcal{f}}$. 
Let $\mathscr{F}=\left\{m_{x, x^{\prime}}\right\}_{x \in E, x^{\prime} \in E^{\prime}}$ be a spectral family on $Z, U$ an open subset of $Z$ which contains the support of every measure in $\mathscr{F}$, and $f$ a mapping of $U$ into a locally compact space $Y$ which is $m_{x, x^{\prime}}$-measurable for every $m_{x, x^{\prime}}$ in $\mathscr{F}$. For each $x \in E, x^{\prime} \in E^{\prime}$, define $m_{x, x^{\prime}}^{f}=f \cdot\left(m_{x, x^{\prime}}\right.$ restricted to $\left.U\right)$. Then $\mathscr{F}^{f}$ $=\left\{m_{x, x^{\prime}}^{f}\right\}_{x \in E, x^{\prime} \in E^{\prime}}$ is a spectral family on $Y$ and $U_{\mathscr{F}_{f, g}}=U_{\mathscr{F},(g \circ f)}$, for all $g \in B^{\infty}(Y)$, where $(g \circ f)^{\prime}(z)=g(f(x))$ if $z \in U$ and $=0$ if $z \notin U$. $\mathscr{F}^{f}$ is called the image of $\mathscr{F}$ by $f$.

We shall say that an element $T$ of $L(E, E)$ commutes with $\mathscr{F}$ if $T U_{\mathscr{F}, f}=U_{\mathscr{F}, f} T$ for every $f \in B^{\infty}(Z)$. An operator $T \in L(E, E)$ is said to be a spectral operator if there is a spectral family $\mathscr{F}_{T}$ on $C$ such that

$$
\begin{aligned}
& T \text { commutes with } \mathscr{F}_{T} ; \\
& \operatorname{sp}\left(T_{\sigma}\right) \subseteq \sigma \text { for every compact } \sigma \subset C .
\end{aligned}
$$

(For each compact $\sigma$, we denote by $T_{\sigma}$ the mapping $x \rightarrow T x$ of $E_{\sigma} \rightarrow E_{\sigma}$, where $E_{\sigma}=P(\sigma) E$.)

It can be shown that the spectral family $\mathscr{F}_{T}$ associated with a spectral operator $T$ is unique. The spectral measure $P_{\mathscr{F}_{T}}$ is sometimes called a resolution of the identity for $T$.

We say that an operator $S \in L(E, E)$ is scalar if there is a spectral family $\mathscr{F}=\left\{m_{x, x^{\prime}}\right\}_{x \in E, x^{\prime} \in E^{\prime}}$ on $C$ of measures with compact support such that $\int_{C^{2}} z d m_{x, x^{\prime}}(z)=\left\langle S x, x^{\prime}\right\rangle$ for every $x \in E, x^{\prime} \in E^{\prime}$. In this case, we write $S=\int_{C} z d P_{\mathscr{F}}(z)$. See [11] concerning the above concepts and definitions.

3. The perturbation theorem. The basic perturbation theorem is given first, followed by some consequences which are more evidently generalizations of the theorems of Bade and Foguel.

THEOREM 1. Let $P$ be a spectral measure on a locally compact space $Z$ with associated spectral family $\mathscr{F}=\left\{m_{x, x^{\prime}}\right\}_{x \in E, x^{\prime} \in E^{\prime}}$. Let $\left\{f_{n}\right\}$ be a sequence of functions in $B(Z)$, and $f$ a complex-valued function defined on $Z$, such that $f_{n}$ converges to $f$ in $m_{x, x^{\prime}}-$ measure for every $x \in E, x^{\prime} \in E^{\prime}$. Let $g \in B^{\infty}(C)$ be such that its discontinuities are contained in a closed set $\alpha$. If $x_{0} \in E$ has the property that $P\left(f^{-1}(\alpha)\right) x_{0}=0$, then

$$
\lim _{n \rightarrow \infty}\left(\int_{z} g \cdot f_{n} d P\right) x_{0}=\left(\int_{z} g \cdot f d P\right) x_{0} .
$$

Proof. Using a theorem of Grothendieck ([9, Theorem 2, p. 146], see also [6, p. 550-551]), it is possible, for every $x_{0}$ in $E$ and every equicontinuous set $A$ in $E^{\prime}$, to find $x_{0}^{\prime} \in E^{\prime}$ such that $m_{x_{0}, x_{0}^{\prime}}$ is positive and the measures $m_{x_{0}, x^{\prime}}, x^{\prime} \in A$, are uniformly absolutely continuous with respect to $m_{x_{0}, x_{0}^{\prime}}$. This means that for each $\varepsilon>0$ there is $\delta_{\varepsilon}>0$ such that $m_{x_{0}, x_{0}^{\prime}}(\sigma)<\delta_{\varepsilon}$ implies $m_{x_{0}, x^{\prime}}(\sigma)<\varepsilon$ for every $x^{\prime} \in A$. 
Let $G_{n}=\int_{\mathrm{z}} g \cdot f_{n} d P$ and $G=\int_{\mathrm{z}} g \cdot f d P$. Then for each $x \in E, x^{\prime} \in E^{\prime}, \varepsilon>0$,

$$
\left|\left\langle\left(G_{n}-G\right) x, x^{\prime}\right\rangle\right|=\left|\int_{Z}\left(g \cdot f_{n}-g \cdot f\right) d m_{x, x^{\prime}}\right|
$$

$$
\leqq \int_{z_{\varepsilon, n}}\left|g \cdot f_{n}-g \cdot f\right| d\left|m_{x, x^{\prime}}\right|+\int_{\mathbf{C z}_{\varepsilon, n}}\left|g \cdot f_{n}-g \cdot f\right| d\left|m_{x, x^{\prime}}\right|
$$

where

$$
Z_{\varepsilon, n}=\left\{z:\left|\left(g \cdot f_{n}\right)(z)-(g \cdot f)(z)\right| \geqq \varepsilon\right\} .
$$

We now fix $\varepsilon>0, A$ an equicontinuous set in $E^{\prime}$, and let $x_{0}$ be as described in the statement of the theorem. Using Grothendieck's work to find $x_{0}^{\prime}$, we have by assumption that $f_{n} \rightarrow f$ in $m_{x_{0}, x}$, -measure. Hence there is a set $\sigma_{0}$ in $Z$ and a subsequence $\left\{f_{n_{i}}\right\} \subseteq\left\{f_{n}\right\}$ such that $m_{x_{0}, x_{0}}\left(\sigma_{0}\right)=0$ and $f_{n_{i}}(z) \rightarrow f(z)$ for all $z \notin \sigma_{0}$. By the continuity of $g,\left(g \cdot f_{n_{i}}\right)(z) \rightarrow(g \cdot f)(z)$ for all $z \notin \sigma_{0} \cup f^{-1}(\alpha)$. Since $P\left(f^{-1}(\alpha)\right) x=0$, we have $m_{x_{0}, x_{0}^{\prime}}\left(\sigma_{0} \cup f^{-1}(\alpha)\right)=0$. Hence we may choose $N$ large enough so that $m_{x_{0}, x_{0}^{\prime}}\left(Z_{\varepsilon, n_{i}}\right) \leqq \delta_{\varepsilon}$ for all $n_{i} \geqq N$. Combining this with (8) above, letting $K$ be the bound of $g$, we get

$$
\begin{aligned}
\left|\left\langle\left(G_{n_{i}}-G\right) x_{0}, x\right\rangle\right| & \leqq 2 K\left|m_{x_{0}, x^{\prime}}\right|\left(Z_{\varepsilon, n_{i}}\right)+\varepsilon\left|m_{x_{0}, x^{\prime}}\right|(Z) \\
& \leqq \varepsilon(2 K+L)
\end{aligned}
$$

for all $x^{\prime} \in A, n_{i} \geqq N$. Here we have let $L=\sup _{x^{\prime} \in A}\left|m_{x_{0}, x^{\prime}}\right|(Z)$. Hence $\left\langle G_{n_{i}} x_{0}, x^{\prime}\right\rangle \rightarrow\left\langle G x_{0}, x^{\prime}\right\rangle$ uniformly for $x^{\prime} \in A$. The same argument may be applied to any subsequence so that $\left\langle G_{n} x_{0}, x^{\prime}\right\rangle \rightarrow\left\langle G x_{0}, x^{\prime}\right\rangle$ uniformly for $x^{\prime} \in A$. Since $A$ is arbitrary and polars of sets in $\mathscr{E}$ generate the topology of $E, G_{n} x_{0} \rightarrow G x_{0}$ and (7) is proved.

TheOREM 2. Let $P_{1}$ be a spectral measure on a locally compact space $Z$, and $P_{2}$ a spectral measure on $C$. Let $\mathscr{F}_{1}=\left\{m_{x, x^{\prime}}^{1}\right\}_{x \in E, x^{\prime} \in E^{\prime}}$ and $\mathscr{F}_{2}=\left\{m_{x, x^{\prime}}^{2}\right\}_{x \in E, x^{\prime} \in E^{\prime}}$ be their respective spectral families of measures on $Z$ and $C$. We suppose also that each $m_{x, x}^{2}$, has compact support. If the range of $P_{1}$ contains the range of $P_{2}$, then there is a function $f$ in $B_{0}(Z)$ such that $\mathscr{F}_{1}^{f}=\mathscr{F}_{2}$.

Proof. For each positive integer $n$, let $\pi_{n}=\left\{\sigma_{i}^{n}, \lambda_{i}^{(n)}\right\}$ be a partition of Supp $\mathscr{F}_{2}$, of norm $\left|\pi_{n}\right| \leqq 1 / n$, with sets of $S_{0}(C)$. That is, for each $n$,

$$
\begin{gathered}
\sigma_{i}^{n} \cap \sigma_{j}^{n}=\varnothing, \quad i \neq j, \\
\bigcup_{i=1}^{\infty} \sigma_{i}^{n}=\operatorname{Supp} \mathscr{F}_{2},
\end{gathered}
$$

$$
\text { diameter } \sigma_{i}^{n} \leqq 1 / n \quad \text { for every } i \text {, }
$$




$$
\text { every } \sigma_{i}^{n} \in S_{0}(C) \text {, and }
$$

$$
\lambda_{i}^{(n)} \in \sigma_{i}^{n} \quad \text { for every } i \text {. }
$$

We can and do suppose further that $m>n$ implies that $\pi_{m}$ is finer than $\pi_{n}$. Hence

$$
\text { for each } m, n, m>n \text {, given any } \sigma_{i}^{m} \text { there }
$$

is a (unique) set $\sigma_{j_{i}}^{n}$ such that $\sigma_{i}^{m} \subseteq \sigma_{j_{i}}^{n}$.

Since both $P_{1}$ and $P_{2}$ are spectral measures, it is easy to verify (for arbitrary $B, B^{\prime} \in S_{0}(z)$ and $\left.\sigma, \sigma^{\prime} \in S_{0}(C)\right)$ that

$$
P_{1}(B)=P_{2}(\sigma) \text { if and only if } P_{1}(\mathcal{C} B)=P_{2}(\mathcal{C} \sigma),
$$

and

$$
\begin{aligned}
& \text { if } \sigma \subset \sigma^{\prime}, P_{1}(B)=P_{2}(\sigma) \text {, and } P_{1}\left(B^{\prime}\right)=P_{2}\left(\sigma^{\prime}\right), \\
& \text { then } P_{1}\left(B \cap B^{\prime}\right)=P_{2}(\sigma) \text {. }
\end{aligned}
$$

The range of $P_{2}$ being contained in the range of $P_{1}$, we may associate with each $\sigma_{i}^{n}$ a set $B_{i}^{n} \in S_{0}(Z)$ such that $P_{1}\left(B_{i}^{n}\right)=P_{2}\left(\sigma_{i}^{n}\right)$ for all $i$ and $n$. From (14) and (16) we may choose the $B_{i}^{n}$ so that for any $m, n, m>n$, we have

$$
\text { for each } B_{i}^{m} \text { there is a (unique) set } B_{j_{i}}^{n} \text { such that }
$$$$
B_{i}^{m} \subset B_{j_{i}}^{n} \text {. Moreover, } B_{i}^{m} \subset B_{j}^{n} \text { if and only if } \sigma_{i}^{m} \subset \sigma_{j}^{n} \text {. }
$$

We now disjoint the sequences $\left\{B_{i}^{n}\right\}$ by defining

$$
D_{1}^{n}=B_{1}^{n} \text { and } D_{i}^{n}=B_{i}^{n} \cap \mathrm{C} \bigcup_{j=1}^{i-1} D_{j}^{n}, \quad i=2,3, \cdots,
$$

for each $n=1,2, \cdots$. Then $D_{i}^{n} \cap D_{j}^{n}=\varnothing$ for $i \neq j$. A simple inductive argument, using (15), shows that $P_{1}\left(D_{i}^{n}\right)=P_{2}\left(\sigma_{i}^{n}\right)$ for all $i$ and $n$. Also, the sets $D_{i}^{n}$ enjoy the same property (17) that the $B_{i}^{n}$ have. Finally, $P_{1}\left(\bigcup_{i=1}^{\infty} D_{i}^{n}\right)=I$ so that if $D_{0}=\mathrm{C} \bigcap_{n=1}^{\infty} \bigcup_{i=1}^{\infty} D_{i}^{n}$, then $P_{1}\left(D_{0}\right)=0$.

We now define

$$
f_{n}(z)=\sum_{i=1}^{\infty} \lambda_{i}^{(n)} \phi_{D_{i}} n(z)
$$

Then $\lim _{n} f_{n}(z)=0$ for all $z \in D_{0}$. For $z \notin D_{0}, m>n$, it follows from (17) that $z \in D_{i}^{m} \cap D_{j}^{n}$ for just one $i$ and one $j=j_{i}$. Hence, by (11) above,

$$
\left|f_{m}(z)-f_{n}(z)\right|=\left|\lambda_{i}^{(m)}-\lambda_{j}^{(n)}\right| \leqq 1 / n,
$$

independent of $z \in G D_{0}$. Thus $\left\{f_{n}(z)\right\}$ is a uniformly Cauchy sequence and converges uniformly on $C D_{0}$ to a function $f \in B_{0}(Z)$.

Using Theorem 1 , for every $g \in \mathscr{K}(C)$, and every $x \in E$, we have 


$$
\lim _{n}\left(\int_{Z} g \cdot f_{n} d P\right) x=\left(\int_{z} g \cdot f d P\right) x .
$$

We now define $g_{n}(\lambda)=\sum_{i=1}^{\infty} g\left(\lambda_{i}^{(n)}\right) \phi_{\sigma_{i}}(\lambda)$. Then

$$
\int_{C} g_{n} d P_{2}=\int_{z} g \cdot f_{n} d P_{1} \quad \text { for each } n .
$$

Moreover, $g_{n} \rightarrow g$ uniformly on Supp $m_{x, x^{\prime}}^{2}$ for each $x \in E, x^{\prime} \in E^{\prime}$. Hence,

$$
\lim _{n} \int_{C} g_{n} d m_{x, x^{\prime}}^{2}=\int_{C} g d m_{x, x^{\prime}}^{2}
$$

Combining (18), (19), and (20), we have

$$
\int_{Z} g \cdot f d m_{x, x^{\prime}}^{1}=\int_{C} g d m_{x, x^{\prime}}^{2} \quad \text { for all } g \in \mathscr{K}(C) .
$$

But this is all we need.

THEOREM 3. Let $\left\{S_{n}\right\}$ be a sequence of scalar operators which converge strongly to a scalar operator $S_{0}$. Let $\mathscr{F}_{n}=\left\{m_{x, x^{\prime}}^{n}\right\}_{x \in E, x^{\prime} \in E^{\prime}}$ be the corresponding spectral families of measures on $C$, and $P_{n}$ the corresponding spectral measures, $n=0,1,2, \cdots$. Suppose there is a spectral measure $P$ on a locally compact space $Z$, with spectral family $\mathscr{F}=\left\{m_{x, x^{\prime}}^{P}\right\}_{x \in E, x^{\prime} \in E^{\prime}}$, such that the range of $P$ contains the range of every $P_{n}$. Let $g \in B_{0}^{\infty}(C)$ have its discontinuities in the closed set $\alpha$. Then there are functions $f_{n}$ in $B_{0}(Z)$ such that

$$
\left\langle S_{n} x, x^{\prime}\right\rangle=\int_{z} f_{n} d m_{x, x^{\prime}}^{P} \text { for } n=0,1,2, \cdots
$$

and

$$
f_{n} \rightarrow f_{0} \text { in } m_{x, x^{\prime}}^{P} \text {-measure for every } x \in E, x^{\prime} \in E^{\prime} .
$$

Moreover,

if, for some $x_{0} \in E, P_{0}(\alpha) x_{0}=0$, then $g\left(S_{n}\right) x_{0} \rightarrow g\left(S_{0}\right) x_{0}$;

if, for some $x_{0} \in E$ and some $\beta \in S(C)$, we have

$P_{0}$ (boundary $\left.\beta\right) x_{0}=0$, then $P_{n}(\beta) x_{0} \rightarrow P_{0}(\beta) x_{0}$;

and finally,

$$
\begin{aligned}
& \text { if } P_{0}(\alpha)=0 \text {, then } g\left(S_{0}\right) \text { is the strong limit of } \\
& g\left(S_{n}\right) .\left(B y g\left(S_{n}\right) \text {, of course, is meant } \int_{C} g d P_{n}\right. \text {.) }
\end{aligned}
$$

Proof. Statement (22) is an immediate result of the previous theorem. 
For $\left(23^{-}\right.$, let $B=\left\{g \in \mathscr{K}(Z): \sup _{z \in Z}|g(z)| \leqq 1\right\}$. Then, for each $x \in E, x^{\prime} \in E^{\prime}$,

$$
\begin{aligned}
\int_{Z}\left|f_{n}-f\right| d\left|m_{x, x^{\prime}}^{P}\right| & =\sup _{g \in B}\left|\int_{Z} g\left(f_{n}-f\right) d m_{x, x^{\prime}}^{P}\right| \\
& =\sup _{g \in B}\left|\int_{Z}\left(f_{n}-f\right) d m_{x, t^{t} U_{F}, x^{\prime}}^{P}\right| \\
& =\sup _{g \in B}\left|\left\langle\left(S_{n}-S_{0}\right) x,{ }^{t} U_{\mathscr{F}, g} x^{\prime}\right\rangle\right| \rightarrow 0
\end{aligned}
$$

as $n \rightarrow \infty$, because $S_{n} \rightarrow S_{0}$ strongly and $\left\{{ }^{t} U_{\mathscr{F}, g} x^{\prime}: g \in B\right\}$ is an equicontinuous set for each $x^{\prime} \in E^{\prime}$.

Statements (24) and (26) follow from (23) and Theorem 1.

Statement (25) follows from (24) by letting $g=\phi_{\beta}$.

COROLLARY. Let $S_{n}$ be a sequence of scalar operators, and $P_{n}$ the corresponding spectral measures on $C, n=1,2, \cdots$. Suppose there is a spectral measure $P$ on a locally compact space $Z$, with spectral family $\mathscr{F}=\left\{m_{x, x^{\prime}}^{P}\right\}_{x \in E, x^{\prime} \in E^{\prime}}$ whose range contains the range of every $P_{n}$. If the sequence $\left\{f_{n}\right\}$ constructed as in

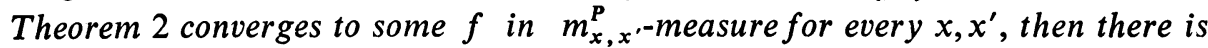
an element $S=\int_{z} f d P$ in $L(E, E)$ such that $\left\langle S_{n} x, x^{\prime}\right\rangle \rightarrow\left\langle S x, x^{\prime}\right\rangle$ for every $x, x^{\prime}$. If also each measure $f \cdot m_{x, x}^{P}$, has compact support, then $S$ is scalar.

In itself this rather complicated corollary does not say much more than the previous theorem. However it points to the connection with Foguel's results as follows. When $E$ is a Banach space and $S_{n}$ is a sequence converging strongly to an operator $S$ then the sequence $\left\{S_{n}\right\}$ is bounded and all the measures involved will have compact support. From the convergence of $S_{n}$ to $S$ we can derive the existence of a function $f$ to which the $f_{n}$ converge in measure. All that is lacking is the spectral measure $P$. But this is derived from Theorem 4 below provided $E$ is weakly complete and the B.A. generated by the ranges of the $P_{n}$ is bounded.

It should be observed that these results may also be sharpened in ordered vector spaces. Such material may be found in [13], where Theorem 6 (p. 154) is clearly related to the above work.

4. Boolean algebras of projections. In this section the connection between Theorem 3 above and Foguel's work is completed. In the course of this development a number of theorems of Bade's are extended to locally convex spaces. A B.A. of projections will mean a subset $\mathfrak{B}$ of $L(E, E)$ consisting of commuting idempotents, including $I$ and 0 , which is a B.A. under the Boolean operations; $P \vee Q=P+Q-P Q$ and $P \wedge Q=P Q$.

DEFINITION 1. We will say that a locally convex space is weakly complete if whenever we have a sequence $\left\{x_{n}\right\}$ of elements of $E$ for which $\lim _{n}\left\langle x_{n}, x^{\prime}\right\rangle$ exists for every $x^{\prime} \in E^{\prime}$, then there is an element $x \in E$ such that $\lim _{n}\left\langle x_{n}, x^{\prime}\right\rangle=\left\langle x, x^{\prime}\right\rangle$ for all $x^{\prime} \in E^{\prime}$. 
THEOREM 4. Let $E$ be weakly complete, and let $\mathfrak{B}$ be a bounded B.A. of projections on $E$. Then there is a compact space $Z$ and a spectral measure $P$ on $Z$ whose range contains $\mathfrak{B}$.

Proof. Let $Z$ be the Stone representation space of $\mathfrak{B}$, and let $\Sigma$ be the field of all open-closed subsets of $Z$. Let $P_{1}: \Sigma \rightarrow \mathfrak{B}$ be the canonical isomorphism between $\Sigma$ and $\mathfrak{B}$. For each $x \in E, x^{\prime} \in E^{\prime}$, define a set function $m_{x, x}$ on $\Sigma$ by the equation

$$
m_{x, x},(\sigma)=\left\langle P_{1}(\sigma) x, x^{\prime}\right\rangle, \quad \sigma \in \Sigma .
$$

It is immediate that each such $m_{x, x}$, is finitely additive and bounded, using the properties of $\mathfrak{B}$. Since every set of $\Sigma$ is both open and closed, $m_{x, x}$, is regular. Hence, as in [5, Chapter III], there is a unique countably additive extension of each $m_{x, x^{\prime}}$, which we denote by the same symbol, to the $\sigma$-field $S_{0}(Z)$ generated by $\Sigma$. If we can show that $\mathscr{F}=\left\{m_{x, x^{\prime}}\right\}$ is a spectral family on $Z$, then its associated spectral measure $P$ will have the properties we need.

We first show that $\mathscr{F}$ is semispectral. Since $m_{\alpha x_{1}+\beta x_{2}, x^{\prime}}(\sigma)=\alpha m_{x_{1}, x^{\prime}}(\sigma)+\beta m_{x_{2}, x^{\prime}}(\sigma)$ and $m_{x, \gamma x_{1}^{\prime}+\delta x_{2}^{\prime}}(\sigma)=\gamma m_{x, x_{1}^{\prime}}(\sigma)+\delta m_{x, x_{2}^{\prime}}(\sigma)$ for all $\sigma \in \Sigma$, and all scalars $\alpha, \beta, \gamma$, and $\delta$, it follows that the mapping $\left(x, x^{\prime}\right) \rightarrow m_{x, x^{\prime}}$ is bilinear.

Next, using a technique taken from [10], we show that the set $\mathfrak{U}_{1}=\left\{\Sigma_{A \in \mathscr{D}} \alpha_{A} P_{1}(A): \mathscr{D}\right.$ a finite partititon of $Z$ by sets of $\left.\Sigma, \sup _{A \in \mathscr{D}}\left|\alpha_{A}\right| \leqq 1\right\}$ is equicontinuous. To see this, we note that for $V$ a convex, balanced, closed neighborhood of zero in $E$, there is a family $\left\{x_{j}^{\prime}\right\}_{j \in J}$ such that

$$
V=\bigcap_{j \in J}\left\{x:\left|\left\langle x, x_{j}^{\prime}\right\rangle\right| \leqq 1\right\} \text {. }
$$

Since $E$ is barelled and $\mathfrak{B}$ is bounded, the set $\left\{P_{1}(\sigma): \sigma \in \Sigma\right\}$ is equicontinuous. Therefore there is a neighborhood $U$ of zero such that $x \in U$ implies $P_{1}(\sigma) x \in V$ for all $\sigma \in \Sigma$. Now, given an element of $\mathfrak{A}_{1}$, let $\mathscr{D}^{\prime}=\left\{A \in \mathscr{D}: \operatorname{Re}\left\langle P_{1}(A) x, x_{j}^{\prime}\right\rangle \geqq 0\right\}$. Then, for each $x \in U$, and $j \in J$,

$$
\left|\sum_{A \in \mathscr{D}^{\prime}} \alpha_{A} \operatorname{Re}\left\langle P_{1}(A) x, x_{j}^{\prime}\right\rangle\right| \leqq \operatorname{Re}\left\langle P_{1}\left(\bigcup_{A \in \mathscr{D}^{\prime}} A\right) x, x_{j}^{\prime}\right\rangle \leqq 1 .
$$

Treating negative real, positive imaginary, and negative imaginary parts in a similar fashion, we get $\left|\left\langle\Sigma_{A \in \mathscr{D}} \alpha_{A} P_{1}(A) x, x_{j}^{\prime}\right\rangle\right| \leqq 4$ for all $j \in J$ and $x \in U$. Thus $\Sigma_{A \in \mathscr{Q}} \alpha_{A} P_{1}(A) x \in 4 V$ if $x \in U$, and $\mathfrak{A}_{1}$ is equicontinuous.

Let $\mathfrak{A}$ be the algebra of functions $f: Z \rightarrow C$ of the form $f(z)=\sum_{i=1}^{n} \alpha_{i} \phi_{\sigma_{i}}(z)$, with the $\alpha_{i}$ being scalars and $\left\{\sigma_{i}\right\}$ a finite disjoint partition of $Z$ by sets of $\Sigma$. For each such $f$, we define $U_{f} \in L(E, E)$ by the equation $U_{f}=\sum_{i=1}^{n} \alpha_{i} P_{1}\left(\sigma_{i}\right)$. It then follows, using the equicontinuity of $\mathfrak{A}_{1}$ proved in the previous paragraph, that

$$
B_{x}=\left\{U_{f} x: f \in \mathfrak{A}_{1}\right\}=\left\{U_{f} x: f \in \mathfrak{U}, \sup |f(z)| \leqq 1\right\}
$$

is bounded for each $x$ in $E$. Also, for each equicontinuous set $A$ in $E^{\prime}$, the set 


$$
\left\{{ }^{t} U_{f} x^{\prime}: f \in \mathfrak{A}, \sup |f(z)| \leqq 1 ; x^{\prime} \in A\right\}
$$

is an equicontinuous subset of $E^{\prime}$.

We observe next that $\mathfrak{A}$ is dense in $\mathscr{K}(Z)$. To see this we note first that since the sets $\sigma_{i}$ are open-closed, every $f$ in $\mathfrak{A}$ is continuous. It is obvious then that $\mathfrak{A}$ is a subalgebra of $\mathscr{K}(Z)$, contains the identity, and contains $\bar{f}$ whenever it contains $f$. Finally, since the sets of $\Sigma$ contain a base for the topology of $Z$, the functions in $\mathfrak{A}$ separate points, and by the Stone-Weierstrass Theorem, $\overline{\mathfrak{A}}=\mathscr{K}(Z)$. From this it follows that for each $x \in E, x^{\prime} \in E^{\prime}$,

$$
\left\|m_{x, x^{\prime}}\right\|=\sup _{\boldsymbol{f} \in \mathfrak{\Re}_{1}}\left|\left\langle U_{\boldsymbol{f}} x, x^{\prime}\right\rangle\right| .
$$

Using (27), for any $\varepsilon>0$, let $V_{x, \varepsilon}$ be the neighborhood of zero in $E^{\prime}$ defined by

$$
V_{x, \varepsilon}=\left\{x^{\prime} \in E^{\prime}:\left|\left\langle y, x^{\prime}\right\rangle\right| \leqq \varepsilon \text { for all } y \in B_{x}\right\} .
$$

Hence, from (29), $\left\|m_{x, x^{\prime}}\right\| \leqq \varepsilon$ for all $x^{\prime} \in V_{x, \ell}$, which shows that the mapping from $E^{\prime}$ to $\mathfrak{M}^{1}(Z)$ defined by $\left(x, x^{\prime}\right) \rightarrow m_{x, x^{\prime}}$ is continuous for each fixed $x$.

Similarly, from (28), we see that for each $\varepsilon>0$ and each equicontinuous set $A$ in $E^{\prime}$, there is a neighborhood of zero in $E, V_{A, \varepsilon}$, such that

$$
\left|\left\langle y,{ }^{t} U_{f} x\right\rangle\right| \leqq \varepsilon \quad \text { for all } f \in \mathfrak{U}_{1}, x^{\prime} \in A .
$$

Since $A$ is arbitrary, it follows, again using (18), that the mapping $\left(x, x^{\prime}\right) \rightarrow m_{x, x^{\prime}}$ is $\mathscr{E}$-hypocontinuous, and, by letting $A=\left\{x^{\prime}\right\}$, continuous from $E$ to $\mathfrak{M}^{1}(Z)$ for each $x^{\prime}$. Hence the family $\mathscr{F}$ is semispectral.

Since $B^{\infty}(Z) \subseteq L^{\infty}(\mathscr{F})$, it follows from remarks in the section on preliminary concepts that whenever one has a semispectral family $\mathscr{F}$, then for every $f \in B^{\infty}(Z)$, there is a linear mapping, which we denote by $\hat{O}_{f}$, from $E$ to $E^{\prime \prime}$ satisfying $\left\langle\hat{U}_{f} x, x^{\prime}\right\rangle=\int_{z} f d m_{x, x^{\prime}}$ for every $x \in E, x^{\prime} \in E^{\prime}$. Moreover, this mapping is continuous if $E^{\prime \prime}$ is given the topology of uniform convergence on sets of $\mathscr{E}$. If we denote by $x \rightarrow \hat{x}$ the canonical embedding of $E$ in $E^{\prime \prime}$, it is evident that for each $f \in \mathfrak{A}$, and each $x^{\prime} \in E^{\prime},\left\langle\hat{U}_{f} x, x^{\prime}\right\rangle=\left\langle\left[U_{f} x\right]^{\wedge}, x^{\prime}\right\rangle$. Since $\mathfrak{A}$ is dense in $\mathscr{K}(Z)$, for each $f \in B_{0}^{\infty}(Z)$, there is a sequence $\left\{f_{n}\right\} \subset \mathfrak{A}$ such that

$$
\lim _{n} m_{x, x^{\prime}}\left(f_{n}\right)=m_{x, x^{\prime}}(f) \text {. }
$$

Hence $\lim _{n}\left\langle U_{f_{n}} x, x^{\prime}\right\rangle$ exists for every $x \in E, x^{\prime} \in E^{\prime}$. Since $E$ is weakly complete, there is an element of $E$ (necessarily unique and well defined independent of the choice of the sequence $f_{n} \rightarrow f$, by virtue of the next equation) which we denote $U_{f} x$, such that

$$
\lim _{n}\left\langle U_{f_{n}} x, x^{\prime}\right\rangle=\left\langle U_{f} x, x^{\prime}\right\rangle .
$$

Hence $\left[U_{f} x\right]^{\wedge}=\hat{U}_{f} x$ for every $x \in E, f \in B_{0}^{\infty}(Z)$. Since polars of sets in $\mathscr{E}$ generate the topology of $E, U_{f} \in L(E, E)$. 
Finally, for every $f$ and $g \in \mathfrak{A}$, supposing

then

$$
f=\sum_{i=1}^{n} \alpha_{i} \phi_{\sigma_{i}} \text { and } g=\sum_{j=1}^{m} \beta_{j} \phi_{\sigma_{j}},
$$

$$
\begin{aligned}
U_{f} U_{g} x & =\sum_{i=1}^{n} \alpha_{i} P\left(\sigma_{i}\right) \sum_{j=1}^{m} \beta_{j} P\left(\sigma_{j}\right) x \\
& =\sum_{i, j=1}^{n, m} \alpha_{i} \beta_{j} P\left(\sigma_{i} \cap \sigma_{j}\right) x=U_{f g} x .
\end{aligned}
$$

Again using the fact that $\mathfrak{A}$ is dense in $\mathscr{K}(Z)$ we find that $U_{f} U_{g}=U_{f g}$ for every $f$ and $g \in B_{0}^{\infty}(Z)$. This completes the proof that $\mathscr{F}$ is spectral.

Following Bade [2], we give the following:

Definition 2. A B.A. $\mathfrak{B}$ of projections on $E$ is said to be complete ( $\sigma$-complete) if for each subset (subsequence) $\left\{P_{\alpha}\right\} \subset \mathfrak{B}, E$ may be decomposed into the (topological) direct sum $E=\mathfrak{M} \oplus \mathfrak{N}$, where

$$
\mathfrak{M}=\operatorname{clm}\left({ }^{2}\right)\left\{P_{\alpha}(E)\right\}, \quad \mathfrak{N}=\bigcap_{\alpha}\left(I-P_{\alpha}\right) E,
$$

and the projection $P_{0}$ which maps $E$ onto $\mathfrak{M}$ and $\mathfrak{N}$ into 0 , is in $\mathfrak{B}$.

THEOREM 5. Let $\mathfrak{B}$ be a bounded complete B.A. of projections on $E$, and $\left\{P_{a}\right\}$ an increasing (decreasing) net in $\mathfrak{B}$. Then

$$
\lim _{\alpha} P_{\alpha} x=\left({ }_{\alpha} \bigvee P_{\alpha}\right) x \quad \text { for all } x \in E \text {. }
$$

If $\mathfrak{B}$ is $\sigma$-complete and bounded, corresponding results are true for sequences.

Proof. Let $\left\{P_{\alpha}\right\}$ be increasing, $P_{0}=\bigvee_{\alpha} P_{\alpha}$. Fix $x \in E$ and let $V$ be a neighborhood of zero in $E$. Find $U$, a neighborhood of zero in $E$, such that $U+U \subset V$. Since $\mathfrak{B}$ is bounded and $E$ is barreled, $\mathfrak{B}$ is equicontinuous and there is $W$, a neighborhood of zero in $E$, such that $P(W) \subset U$ for all $P \in \mathfrak{B}$. Since $P_{0}(E)=\operatorname{clm}\left\{P_{\alpha}(E)\right\}$, we can find $y=\sum_{i=1}^{n} \beta_{i} z_{i}$ such that $y-P_{0} x \in W \cap U$, and each $z_{i} \in P_{\alpha_{i}}(E)$ for some $\alpha_{i}$. Pick $\alpha_{0}$ so that $\alpha_{0} \geqq \alpha_{i}$ for $i=1,2, \cdots, n$. Then for $\alpha \geqq \alpha_{0}, P_{\alpha} y=y$ so that

$$
\begin{aligned}
P_{\alpha} x-P_{0} x & =\left(P_{\alpha} x-y\right)+\left(y-P_{0} x\right) \in P_{\alpha}\left(P_{0} x-y\right)+U \\
& \subseteq U+U \subseteq V .
\end{aligned}
$$

Since $V$ is arbitrary, $\lim _{\alpha} P_{\alpha} x=P_{0} x$. The proof for the decreasing case and for sequences is similar.

This theorem may also be considered to be a corollary of the (relatively easy extension to locally convex spaces of a) theorem of Barry's [3].

(2) clm $=$ smallest closed linear manifold containing \{\} . 
Theorem 6. Let $\mathfrak{B}$ be a B.A. of projections in E. If $\mathfrak{B}$ is bounded and $\sigma$ complete, then it is the range of a spectral measure on its Stone representation space $Z$.

Proof. As usual, let $P$ be the isomorphism of the field $\Sigma$ of open-closed sets of $Z$ onto $\mathfrak{B}$. For each $\sigma \in S_{0}(Z)$ there is $\sigma^{\prime} \in \Sigma$ such that the symmetric difference, $\sigma \Delta \sigma^{\prime}$, is a set of Baire Cat.I. If $\sigma^{\prime \prime}$ is another set of $\Sigma$ such that $\sigma \Delta \sigma^{\prime \prime}$ is Cat.I., then so is $\sigma^{\prime} \Delta \sigma^{\prime \prime}$ of Cat.I. Since both $\sigma^{\prime}$ and $\sigma^{\prime \prime}$ are in $\Sigma$, this means that $\sigma \Delta \sigma^{\prime \prime}$ is open, which is impossible unless $\sigma^{\prime}=\sigma^{\prime \prime}$. Hence there is no inconsistency in defining $P_{1}$ for $\sigma$ in $S_{0}(Z)$ by $P_{1}(\sigma)=P\left(\sigma^{\prime}\right)$. The only point at issue is countable additivity. Let $\left\{\sigma_{n}\right\}$ be a disjoint sequence of sets in $S_{0}(Z),\left\{\sigma_{n}^{\prime}\right\}$ the corresponding sets in $\Sigma$. Using the $\sigma$-completeness of $\mathfrak{B}$ there is a set $\sigma^{\prime} \in \Sigma$ such that $P\left(\sigma^{\prime}\right)=\bigvee_{n} P\left(\sigma_{n}^{\prime}\right)$. Then $\sigma^{\prime} \Delta\left(\bigcup_{n} \sigma_{n}^{\prime}\right)$ is Cat.I. so that $P_{1}\left(\bigcup_{n} \sigma_{n}\right)=P\left(\sigma^{\prime}\right)$. Finally, from the previous theorem, $\left(\bigvee_{n} P\left(\sigma_{n}^{\prime}\right)\right) x=\Sigma_{n}\left(P\left(\sigma_{n}^{\prime}\right) x\right)=\Sigma_{n}\left(P_{1}\left(\sigma_{n}\right) x\right)$ for each $x \in E$.

THEOREM 7. A convex subset of $L(E, E)$ has the same closure in the topology of convergence at each point of $E$ as it does in the topology of convergence for each pair $x \in E, x^{\prime} \in E^{\prime}$. (I.e. in what are referred to as strong and weak operator topologies in the theory of Banach spaces.)

Proof. This is just a repetition of Lemma 3.3 in [1]. The proof given there may be translated into the language of locally convex spaces without serious difficulty, the specific properties of norms being irrelevant here. As an illustration of how the translation may proceed, at one point a Banach space of $n$-tuples with maximum norm is constructed. If the topology of $E$ is generated by seminorms $\left\{p_{\alpha}\right\}$, each such $p_{\alpha}$ may be used to generate a seminorm $\hat{p}_{\alpha}$ on the space of $n$-tuples $\hat{x}=\left[x_{1}, \cdots, x_{n}\right]$ of elements of $E$ by letting $\hat{p}_{2}(\hat{x})=\max _{1 \leqq i \leqq n} p_{\alpha}\left(x_{i}\right)$. These seminorms then generate a locally convex topology on $E \times E \times \cdots \times E$ ( $n$ factors).

THEOREM 8. Let $\mathfrak{B}$ be a bounded complete B.A. of projections on E. Let $\mathfrak{A}$ be the algebra generated by $\mathfrak{B}$ in $L(E, E), \overline{\mathfrak{U}}^{w}$ ( $=\overline{\mathfrak{A}}^{s}$ by the previous theorem), its closure in the weak operator topology. If $T \in \overline{\mathfrak{A}}^{w}$ and if $K$ and $K^{\prime}$ are finite subsets of $E$ such that $T\left(K^{\prime}\right)=0$, then there is $P_{0}=P_{K, K^{\prime}} \in \mathfrak{B}$ such that $T(K) \subseteq P_{0}(E)$ and $K^{\prime} \subseteq\left(I-P_{0}\right)(E)$.

Proof. The theorem asserts the existence of a $P_{0} \in \mathfrak{B}$ such that $P T y=T y$ and $P z=0$ for all $y \in K, z \in K^{\prime}$. Since $T \in \overline{\mathfrak{U}}$, for any neighborhood $V$ of zero in $E$ there is $A_{V} \in \mathfrak{A}$ such that $A_{V} y-T y \in V$ and $A_{V} z \in V$ for all $y \in K, z \in K^{\prime}$. The cardinality of $K$ and $K^{\prime}$ is irrelevant (though it must be finite) from this point on. To simplify the computations we suppose that $K$ and $K^{\prime}$ each contain a single point, $K=\{y\}$ and $K^{\prime}=\{z\}$, and that $\mathfrak{D}$ is a filter base of neighborhoods of zero in $E$ consisting in closed convex balanced sets. The proof is modeled on that 
of Theorem 3.4 in [1]. However, the "translation" is fairly involved and is given with some completeness.

For each $V \in \mathfrak{D}, A_{V}=\sum_{i=1}^{n V} \alpha_{i, V} P\left(\sigma_{i, V}\right)$ where $P$ is the spectral measure whose range is $\mathfrak{B}$, and the $\sigma_{i, V}$ are clopen subsets of the Stone space of $\mathfrak{B}$. For each $\varepsilon>0, V \in \mathcal{D}$, let $\sigma_{V}^{\varepsilon}=\bigcup\left\{\sigma_{i, V}:\left|\alpha_{i, V}\right|>\varepsilon\right\}$. Obviously, if $\varepsilon_{1} \leqq \varepsilon_{2}$, then $P\left(\sigma_{V}^{z_{2}}\right) \leqq P\left(\sigma_{V}^{\varepsilon_{1}}\right)$. Now define

$$
P_{V, \varepsilon}=\bigvee\left\{P\left(\sigma_{W}^{\varepsilon}\right): W \in \mathfrak{D}, W \subseteq V\right\}
$$

Then $\varepsilon_{1} \leqq \varepsilon_{2}$ implies

$$
P_{V, \varepsilon_{2}} \leqq P_{V, \varepsilon_{1}} \text {. }
$$

Likewise, for each $\varepsilon$, if $V_{1} \subseteq V_{2}$, both elements of $\mathcal{D}$, then $P_{V_{1}, \varepsilon} \leqq P_{V_{2}, \varepsilon}$. Consequently if $V_{1} \subseteq V_{2}$ and $\varepsilon_{1} \leqq \varepsilon_{2}$, then $P_{V_{1}, \varepsilon_{2}} P_{V_{2, \varepsilon_{1}}}=P_{V_{1}, \varepsilon_{2}}$. More important, for each $\varepsilon$,

$$
P_{\varepsilon} x=\left(\bigwedge P_{V, \varepsilon}\right) x=\lim _{V} P_{V, \varepsilon} x \quad \text { for all } x \in E .
$$

From (30) we see that $\varepsilon_{1} \leqq \varepsilon_{2}$ implies $P_{\varepsilon_{1}} \geqq P_{\varepsilon_{2}}$. Hence $P_{0} x=\left(\bigvee_{\varepsilon>0} P_{\varepsilon}\right) x=$ $\lim _{\varepsilon \rightarrow 0} P_{\varepsilon} x, x \in E$. Now

$$
P_{V, \varepsilon} T y-T y=P_{V, \varepsilon}\left(T y-A_{V} y\right)+\left(P_{V, \varepsilon} A_{V} y-A_{V} y\right)+\left(A_{V} y-T_{y}\right) .
$$

Thus given $W \in \mathfrak{D}$, we find $U \in \mathfrak{D}$ such that $U+U+U \subseteq W$ and $\beta_{U}>0$ such that $\|f\| \leqq 1$ implies $U_{f} y \in \beta_{U} U$. Since $P_{V, \varepsilon} \geqq P\left(\sigma_{V}^{\varepsilon}\right)$ for all $V$ and $\varepsilon, P_{V, \varepsilon} A_{V}-A_{V}=U_{y}$ for some $y$ satisfying $\|y\| \leqq \varepsilon$. Hence $\left(P_{V .{ }_{e}} A_{V}-A_{V}\right) y \in U$ for all $V$, all $\varepsilon \leqq 1 / \beta_{U}$. By the equicontinuity of $\mathfrak{B}$ we may find $V^{\prime}$ such that $P_{V, \varepsilon}\left(T y-A_{V} y\right) \in U$ and $A_{V} y-T y \in V \subseteq U$ for all $\varepsilon$ and all $V \subseteq V^{\prime}$.

Substituting these results in (31) we find that given $W$, there exists $V^{\prime} \in \mathfrak{D}$ and $\gamma>0\left(\gamma=1 / \beta_{U}\right)$ such that

$$
P_{V, \varepsilon} T y-T y \in W \quad \text { for all } V \subseteq V^{\prime}, \varepsilon \leqq \gamma .
$$

It follows readily that

$$
P_{\varepsilon} T y-T y \in W \text { for all } \varepsilon \leqq \gamma \text {. }
$$

Hence $P_{0} T y-T y \in W$. Since $W$ is arbitrary $P_{0} T y=T y$.

Finally, to show $P_{0} z=0$, we define

$$
A_{V}^{\prime}=\Sigma \beta_{i, V} P\left(\sigma_{i, V}\right)
$$

where $\beta_{i, V}=1 / \alpha_{i, V}$ whenever $\alpha_{i, V} \neq 0$, and $\beta_{i, V}=0$ otherwise. Then

$$
P\left(\sigma_{V}^{\varepsilon}\right) A_{V}^{\prime}=\sum_{\left|\beta_{i}, V\right|<1 / \varepsilon} \beta_{i, V} P\left(\sigma_{i, V}\right) .
$$

Again using the equicontinuity of $\left\{U_{f}:\|f\| \leqq 1\right\}$, given $U \in \mathfrak{D}$ and $\varepsilon>0$ we may find $V$ such that 


$$
P_{V, \delta} z \in \mathrm{I} U \quad \text { for all } \delta \geqq \varepsilon .
$$

Using the equicontinuity of $\mathfrak{B}$ we may assume that there is a $W$ such that (32) holds for all $V \supseteq W$ so that $P_{\delta} z \in U$ for all $\delta \geqq \varepsilon$. Hence $P_{0} z \in U$. Since $U$ is arbitrary $P_{0} z=0$.

This theorem is really just a lemma leading to the next theorem.

THEOREM 9. A bounded complete B.A. of projections contains every projection in the weakly closed algebra it generates.

Proof. As in the first paragraph of the proof of Theorem 3.4 of [1]. The hard part is done above.

THEOREM 10. If $\mathfrak{B}$ is a bounded complete B.A. of projections then $\mathfrak{B}=\overline{\mathfrak{B}}^{\text {s. }}$.

Proof. $T \in \overline{\mathfrak{B}}^{s}$ implies $T \in \overline{\mathfrak{X}}^{w}$ and $T^{2}=T$. Hence $T \in \mathfrak{B}$ by the previous theorem.

The last theorem given here is a generalization of Theorem 2.7 in [2]. We are indebted to Bertram Walsh for a simplification of the hypotheses.

THEOREM 11. Let $E$ be metrizable, $\mathfrak{B}$ a bounded $\sigma$-complete B.A. of projections on $E$. IThen $\overline{\mathfrak{B}^{s}}$ is complete.

Proof. Again the proof is in outline a translation of that given in [2]. The condition of metrizability on $E$ is used to accomplish countable decomposability for the restriction $\mathfrak{B}_{x}$ of $\mathfrak{B}$ to the cyclic subspace

$$
\mathfrak{M}(x)=\operatorname{clm}\{P x: P \in \mathfrak{B}\} \text {. }
$$

\section{BIBLIOGRAPHY}

1. W. G. Bade, Weak and strong limits of spectral operators, Pacific J. Math. 4 (1954), 393-413.

2. - On Boolean algebras of projections and algebras of operators, Trans. Amer. Math. Soc. 80 (1955), 345-360.

3. J. Y. Barry, On the convergence of ordered sets of projections, Proc. Amer. Math. Soc. 5 (1954), 313-314.

4. N. Dunford, Spectral theory. II. Resolutions of the identity, Pacific J. Math. 2 (1952), $559-614$.

5. N. Dunford and J. Schwartz, Linear operators, Interscience, New York, 1958.

6. D. A. Edwards and C. T. Ionescu Tulcea, Some remarks on commutative algebras of operators on Banach spaces, Trans. Amer. Math. Soc. 93 (1959), 541-551.

7. S. R. Foguel, Sums and products of commuting spectral operators, Ark. Mat. 3 (1957), 449-461.

8. - A perturbation theorem for scalar operators, Comm. Pure Appl. Math. 11 (1958)‘ 293-295.

9. A Grothendieck, Sur les applications linéaires faiblement compactes d'espaces du type $C(K)$, Canad. J. Math. 2 (1952), 129-173. 
10. C. T. Ionescu Tulcea, Spectral operators on a locally convex space, (unpublished manuscript).

11. - Spectral operators on locally convex spaces, Bull. Amer. Math. Soc. 67 (1961), $125-128$.

12. F. Rellich, Störungstheorie der Spektralzerlegung. II, Math. Ann. 113 (1936), 677-685.

13. H. Schaeffer, Spectral measures in locally convex algebras, Acta. Math. 107 (1962), 125-173.

MARQUETTE UNIVERSITY,

MiLWAUREe, Wisconsin 